\title{
1 GAD65Cre drives reporter expression in 2 multiple taste cell types
}

3 AUTHORS AND AFFILIATIONS

4 Eric D. Larson*, Aurelie Vandenbeuch*, Catherine B. Anderson, Sue C. Kinnamon

5 Department of Otolaryngology, University of Colorado Anschutz Medical Campus and Rocky

6 Mountain Taste and Smell Center, Aurora, CO

$7 \quad$ * denotes equal contributions

\section{ADDRESS AND EMAIL OF}

10 Correspondence to be sent to:

11 Eric D. Larson

12 University of Colorado Anschutz Medical Campus

13 RC2-Mail stop 8606

$1412700 \mathrm{E}$. 19th avenue

15 Aurora, CO, 80045

16 eric.larson@cuanschutz.edu

18 KEYWORDS

19 Taste, gad65, optogenetics, Type I cells 


\section{ABSTRACT}

21 In taste buds, Type I cells represent the majority of cells (50-60\%) and primarily have a glial-like

22 function in taste buds. However, recent studies suggest that they have additional sensory and

23 signaling functions including amiloride-sensitive salt transduction, oxytocin modulation of taste,

24 and substance P mediated GABA release. Nonetheless, the overall function of Type I cells in

25 transduction and signaling remains unclear, primarily because of the lack of a reliable reporter

26 for this cell type. GAD65 expression is specific to Type I taste cells and GAD65 has been used

27 as a Cre driver to study Type I cells in salt taste transduction. To test the specificity of

28 transgene-driven expression, we crossed GAD65Cre mice with floxed tdTomato and

29 Channelrhodopsin (ChR2) lines and examined the progeny with immunochemistry, chorda

30 tympani recording, and calcium imaging. We report that while many tdTomato+ taste cells

31 express NTPDase2, a specific marker of Type I cells, we see expression of tdTomato in both

32 Gustducin and SNAP25 positive taste cells. We also see ChR2 in cells just outside the

33 fungiform taste buds. Chorda tympani recordings in the GAD65Cre/ChR2 mice show large

34 responses to blue light, larger than any response to standard taste stimuli. Further, several

35 isolated tdTomato positive taste cells responded to $\mathrm{KCl}$ depolarization with increases in 36 intracellular calcium, indicating the presence of voltage-gated calcium channels. Taken

37 together, these data suggest that GAD65Cre mice drive expression in multiple taste cell types

38 and thus cannot be considered a reliable reporter of Type I cell function.

INTRODUCTION

41 It is commonly assumed that taste buds consist of 3 cell types based on morphological,

42 molecular and physiological characteristics(for review, see Roper and Chaudhari, 2017). Each

43 cell type responds to different taste stimuli and communicates in various ways with the afferent

44 nerve fibers to convey the message to the central nervous system. More specifically, Type II

45 cells possess the receptors and downstream transduction components for sweet, umami and

46 bitter stimuli. Although they do not form conventional synapses with nerve fibers, Type II cells

47 release ATP via non-vesicular "channel" synapses to stimulate afferent gustatory nerve

48 fibers(Romanov et al., 2018; Nomura et al., 2020). Conversely, Type III cells, which respond to

49 acid stimuli and some salts, form conventional synapses with nerve fibers and release serotonin 
to activate neighboring cells or nerve fibers. Finally, Type I cells are the least understood cell type, representing $\sim 50 \%$ of the total number of cells in a taste bud (for review, see Roper and Chaudhari, 2017). Because they express NTPDase2 for the degradation of ATP released by other cells (Bartel et al., 2006; Vandenbeuch et al., 2013), and because they wrap around Type II and Type III cells (Yang et al., 2020), Type I cells are thought to have a glial-like function, similar to astrocytes in the nervous system. However, several studies have suggested a more complex function of Type I cells including a potential role in amiloride-sensitive salt taste transduction (Vandenbeuch et al., 2008, Baumer-Harrison et al., 2020), release of oxytocin to modulate taste (Sinclair et al., 2010), and release of GABA in response to trigeminal modulation with substance P (Huang and $\mathrm{Wu}, 2018$ ). In order to examine the potential roles of Type I cells a specific marker is needed to both identify isolated cells for physiology and to manipulate gene expression.

GAD65 is expressed by mature Type I taste cells (Dvoryanchikov et al., 2011) and thus may be a good candidate to use as a Cre driver to report expression in Type I taste cells. GAD65Cre mice are available commercially and have been documented to drive expression specifically in GAD65 expressing neurons in the brain (Taniguchi et al., 2011). Taste cells, unlike neurons, turn over throughout life and since Cre is turned on at the birth of a cell and remains on, it is important to document its specificity of expression in adult taste cells. For example, if GAD65 is expressed transiently in taste cell progenitors, it may drive expression in more than just Type I taste cells. To examine more closely the specificity of expression, we obtained the commercially available GAD65Cre mice and crossed them with floxed Channelrhodopsin (ChR2) or Rosa tdTomato mice to examine specificity of expression. We used immunocytochemistry, chorda tympani nerve recordings, and calcium imaging of isolated tdTomato-positive taste cells. We conclude that while many Type I cells are labeled with either reporter, we show some expression of reporter in Type II and Type III taste cells, suggesting that GAD65 may be transiently expressed in some taste cell progenitors. Thus, this line cannot be used to drive reporter activity faithfully in Type I cells. 
All animal procedures in the study were approved by the Institutional Animal Care and Use Committee at the University of Colorado Anschutz Medical Campus. Genetically altered mice used in the study are described in Table 1. Male and female mice aged $4-8$ months were used for this study. Mice were kept in passive air exchange caging under a 12h light/dark cycle and were provided food and water ad libitum. TRPM5GFP mice are described in (Clapp et al., 2006) and PKD2L1YFP mice are described in (Chang et al., 2010).

\section{Immunohistochemistry}

87 Tissues were fixed by immersion ( $4 \mathrm{~h}$ at $4 \mathrm{C}$ ) or transcardial perfusion followed by post-fixation (4h at $4^{\circ} \mathrm{C}$ ) with $4 \%$ paraformaldehyde dissolved in $0.1 \mathrm{M}$ Phosphate Buffer (PB, pH 7.2). Fixed tissues were cryoprotected overnight at $4^{\circ} \mathrm{C}$ in $20 \%$ sucrose dissolved in $0.1 \mathrm{M} \mathrm{PB}$ prior to embedding in Optimal Cutting Temperature Compound (Tissue-Tek, Torrance, CA). Twelve-16 $\mu \mathrm{m}$ sections were cut using a cryostat (Leica) and collected on charged glass microscope slides (SuperFrost Plus, Fisher Scientific). Slides were stored at $-20^{\circ} \mathrm{C}$ until use. After thawing, slides were rinsed with PBS and incubated in blocking solution ( $2 \%$ normal donkey serum, $1 \%$ bovine serum albumin, $0.3 \%$ triton in PBS) prior to overnight incubation in primary antibody solution. Antisera used in this study are included in Table 2 and were diluted in blocking solution. Secondary antisera were diluted in blocking solution and applied for $3 \mathrm{~h}$ at room temperature. Slides were counterstained with DAPI $(0.5 \mathrm{ug} / \mathrm{mL}$, ThermoFisher) and coverslipped with FluoroMount-G (Southern Biotech).

Whole mount IHC: To image whole mount fungiform taste buds, fungiform epithelia were peeled as described below, pinned flat and fixed in $4 \%$ paraformaldehyde for 1 hour at room temperature. $\mathrm{IHC}$ protocol was like that of tissue sections, but with increased timing: overnight blocking at $4 \mathrm{C}, 48 \mathrm{~h}$ primary antibody at $4 \mathrm{C}$, overnight secondary antibody at $4 \mathrm{C}$. Tissue was mounted on a microscope slide using FluoroMount-G.

Confocal Imaging: All images were acquired using a Leica SP8 laser scanning confocal microscope with a 63x oil immersion objective (NA1.4). Images were acquired with 1024x1024 pixel resolution and axial intervals of $\sim 0.3 \mu \mathrm{m}$. Fluorescent signals were detected sequentially by line to limit any cross-channel detection except for DAPI and Alexa-647 which were acquired simultaneously. Images were processed using a combination of ImageJ (FIJI SCR_002285) and the Adobe CC platform (San Jose, CA). Manipulation of images included only linear 
and pixel density resampling ([Photoshop:Image Size: resample] to achieve appropriate resolution for display).

Image analysis: Cell profile intensities were measured by creating ROls around cell profiles at multiple positions through the Z-stack, with 5-7 um axial spacing between positions (and excluding the first and last 5 um of whole-mount images) using ImageJ. The mean intensity per

118 ROI was calculated for each channel at each axial position. For each image, background values 119 and maximum values were calculated using a maximum z-projection of optical planes used in

120 the analysis above. For background values, 5 ROls were drawn in areas directly adjacent to the 121 taste bud, and the mean pixel intensity values were measured. Threshold for each channel was

122 calculated as mean background plus 2 standard deviations. Maximum values were calculated

123 by determining the pixel intensity value that represented $95.5 \%$ of non-zero pixels, per channel.

124 Measurements from ImageJ were imported to R (R Core Team, 2019) using a custom script 125 where background and cell profile levels were scaled based on the maximum pixel values, summarized, and plotted using ggplot2 (Wickham, 2016).

\section{Taste Cell Isolation}

128 Circumvallate and fungiform taste buds and cells were isolated as previously described (Béhé et 129 al., 1990; Clapp et al., 2006). Mice were euthanized with $\mathrm{CO}_{2}$ followed by cervical dislocation. 130 An enzyme cocktail consisting of $1 \mathrm{mg} / \mathrm{ml}$ collagenase A (Roche, Indianapolis, IN), $3 \mathrm{mg} / \mathrm{ml}$ 131 Dispase II (Roche, Indianapolis, IN), and $1 \mathrm{mg} / \mathrm{ml}$ trypsin inhibitor (Sigma, St. Louis, MO) 132 dissolved in Tyrode's (140 NaCl, $5 \mathrm{KCl}, 1 \mathrm{MgCl}$, $1 \mathrm{CaCl}$, $10 \mathrm{HEPES}, 10$ glucose, and 1 133 pyruvate $(\mathrm{pH} 7.4$ with $\mathrm{NaOH}))$ was injected underneath the epithelium of the tongue. After 134 incubation for $40 \mathrm{~min}$. in oxygenated Ca-Mg free Tyrode's (same as above but $1 \mathrm{mM}$ BAPTA 135 replaced the divalent cations), the epithelium was separated from the underlying connective 136 tissue and placed in Ca-Mg free Tyrode's for 30 min. Taste buds were removed by gentle 137 suction with a fire-polished pipette and plated onto glass coverslips coated with Cell Tak (BD 138 Biosciences, Bedford, MA) or poly-L-lysine (Sigma, St. Louis, MO). Coverslips were placed in a perfusion chamber (RC-25F, Warner Scientific Inc., Hamden, CT). All data for calcium imaging were obtained from isolated taste cells or small cell clusters to verify the presence or absence of tdTomato expression. 


\section{Calcium imaging}

14410 minutes after plating on Cell-Tak coated coverslips, cells were loaded with $2 \mu \mathrm{M}$ fura-2 AM

145 (Invitrogen, Waltham, MA) for 25 minutes at $35^{\circ} \mathrm{C}$. After loading, cells were washed and kept in 146 a continuous flow of Tyrode's using a gravity flow perfusion system (Automate Scientific Inc., 147 San Francisco, CA). Depolarizing concentrations of $\mathrm{KCl}(55 \mathrm{mM})$ were made in Tyrode's (with $14890 \mathrm{mM} \mathrm{NaCl}$ to maintain osmolarity). Sequential excitation at 340 and $380 \mathrm{~nm}$ from an $\mathrm{Hg}$ light 149 source was controlled using a Sutter Lambda 10-3. Emission at $\sim 510 \mathrm{~nm}$ was measured using 150 a Retiga R3 (QImaging) and a 40x oil immersion objective (NA1.4) on an inverted Olympus 151 IX71. Images were captured every 3 seconds using Imaging Workbench 6.1 (Indec Biosystems, 152 Inc). Baseline ratio per cell was measured as the mean ratio 20 seconds pre- and post-stimulus 153 application. In the event a cell did not return to baseline, the first 20 seconds of a stable ratio 154 was used as the post-stimulus baseline. If the post-stimulus baseline was greater than $25 \%$ of 155 the response amplitude, the cell was removed from analysis. A ratio of emission at 510 from 156 excitations at 340 and $380 \mathrm{~nm}$ was considered a response if it fell 2 standard deviations or more 157 above the median baseline ratio across all cells.

\section{Nerve recording}

160 Mice were anesthetized with urethane (2 g/kg; i.p), maintained in a head holder, and a cannula 161 was placed in the trachea to facilitate breathing. The chorda tympani nerve was exposed using 162 a ventral approach, cut near the tympanic bulla and placed on a platinum-iridium wire. A 163 reference electrode was placed in a nearby tissue. The signal was fed to an amplifier (P511; 164 Grass Instruments), integrated, and recorded using AcqKnowledge software (Biopac). The 165 anterior part of the tongue was stimulated with different tastants (applied for $30 \mathrm{~s}$ and then 166 rinsed with water for $40 \mathrm{~s}$ ) with a constant flow pump (Fisher Scientific). Stimuli consisted of (in $167 \mathrm{mM}$ ): $\mathrm{NH}_{4} \mathrm{Cl} 100$, quinine 10 , sucrose $500, \mathrm{NaCl} 100$, citric acid 10. For light stimulation, a LED 168 blue light (470nm) was positioned in close proximity to the tongue. Light pulses were chosen to 169 give an optimal nerve response $(5 \mathrm{~Hz}, 7 \mathrm{~mW}, 50 \%$ duty cycle; Wilson et al., 2019; Vandenbeuch 170 et al., 2020). The amplitude of each integrated response was averaged over the $30 \mathrm{~s}$ application 171 using AcqKnowledge software and subtracted to the average baseline activity averaged $5 \mathrm{~s}$ 172 before tastant application. In order to average responses from different animals, each response was normalized to baseline using the following formula: (amplitude response - baseline) / 
174 baseline)(Vandenbeuch et al., 2015; Larson et al., 2020). A Grubb's test was used to eliminate 175 significant outliers (GraphPad). Responses were compared using a one-way ANOVA with 176 Tukey's post hoc test.

177 RESULTS

\section{Immunohistochemical characterization of GAD65Cre}

179 To use a genetic model to study Type I cell function, the Cre driver allele of interest must be 180 specific to Type I cells in adult mice. Thus, we carefully characterized the specificity of Cre 181 recombinase in GAD65Cre mice using floxed tdTomato and ChR2 reporter alleles. We 182 additionally crossed GAD65Cre/tdTomato with TRPM5GFP and PKD2L1YFP (to label most 183 Type II and III cells with GFP/YFP (Clapp et al., 2006; Chang et al., 2010; Wilson et al., 2017)). 184 GAD65Cre-driven tdTomato and ChR2 were robustly expressed in taste buds of multiple taste 185 fields (including fungiform and CVP (figure 1-4) and foliate and soft palate (not shown). To 186 determine if labeled cells were Type I cells, we labeled lingual sections from 187 GAD65Cre/tdTomato mice with antibodies against NTPDase2, a Type I cell marker (Bartel et 188 al., 2006). NTPDase2-immunoreactivity localizes to Type I cell membranes and often coincided 189 with the tomato reporter, suggesting that tomato+ cells are indeed Type I cells (Fig 1).

190 NTPDase2 immunoreactivity also often colocalized with ChR2 (not shown), which is a 191 membrane-associated reporter, though colocalization of NTPDase2 and ChR2 does not 192 necessarily mean they are in the same cell; signals from two adjacent (abutting) cell membranes would result in artefactual colocalization given resolution limits of light microscopy

194 (Corson and Erisir, 2013; Stratford et al., 2017). Thus, we chose not to pursue quantitative 195 analysis here. Qualitatively, most, but not all, NTPDase2 immunoreactive cells were associated with either tdTomato or ChR2 fluorescence. Conversely, not all tdTomato or ChR2 signals were associated with NTPDase2 immunoreactivity. This suggested that another, unique cell type is labeled with the reporter or that the reporter may be expressed in a subset of Type II and/or Type III cells. Additionally, ChR2+ cells were observed around the circumference of some but not all fungiform taste buds (5/8 taste buds with ChR2, Figure 2). These cells were not immunoreactive to any other markers used in this study. Interestingly, these cells were observed less frequently (and less brightly) when tdTomato was used as the reporter (not shown). Stimulation of ChR2 in these cells has the potential for unforeseen effects. Taken 
204 together, these data support that Cre recombinase is expressed in Type I cells but is not limited

205 to Type I cells.

206

To examine the taste cell type-specificity of GAD65Cre activity further, we examined coexpression of tdTomato with Type II and Type III cell markers, Gustducin and SNAP25, respectively. In 7 whole-mount fungiform taste buds from GAD65Cre/tdTomato mice, 4 had at least 1 cell where above-threshold tdTomato fluorescence was detected in a Gustducinimmunoreactive cell, and 4 had at least 1 cell where above-threshold tdTomato fluorescence was detected in a SNAP25 immunoreactive cell (Figure 3). Quantitative analysis of whole mount

213 fungiform epithelia revealed that of 498 cell profiles analyzed, 20 (4.0\%) had above threshold

214 levels of both tdTomato fluorescence and Gustducin immunoreactivity, and $13(2.6 \%)$ had

215 above threshold levels of tdTomato fluorescence and SNAP25 immunoreactivity. The remaining

216 ROls included 183 (36.7\%) tdTomato+, 170 (34.1\%) Gustducin+, 46 (9.2\%) SNAP25+, and 66

$217(13.3 \%)$ sub-threshold profiles. SNAP25-immunoreactivity and Gustducin-immunoreactivity

218 were never observed in the same profile (not shown). These data suggest that a small

219 proportion of type II and III cells are also labeled by GAD65Cre.

To eliminate any confounds of antibody-labeling and to maximize the number of Type II and III cells identified, we examined taste buds from GAD65Cre/tdTomato/TRPM5GFP/PKD2L1YFP mice. GFP and YFP fluorophores in these tissues were detected using an anti-GFP antibody

224 (cross-reacts with YFP), allowing us to visualize (but not distinguish) Type II and III cells in the same channel. A subset of Type II cells was further identified by Gustducin immunoreactivity. In 8 whole mount fungiform taste buds that were imaged, 6 taste buds had at least one Type II or III cell that coincided with tdTomato (Fig 4). These observations were consistent with antibody labeling in GAD65Cre/tdTomato mice and held true for other taste fields including posterior regions (circumvallate and foliate papilla). We observed tdTomato expression in $13.5 \%$ of fungiform, $3.7 \%$ of soft palate, $5.4 \%$ of foliate, and $5.8 \%$ of circumvallate cells immunoreactive for TRPM5GFP/PKD2L1YFP and/or Gustducin (Table 3).

232 Overall, we hypothesize that the expression of Cre recombinase in more than just Type I cells in

233 these mice could be the result of a shared stem/precursor population of adult taste cells

234 (Castillo et al., 2014; Gaillard et al., 2015). Thus, activation of Cre recombinase could result in

235 the constitutive reporter expression in adult taste cells that no longer express GAD65, as well as

236 in Type I taste cells known to express mRNA coding for GAD65 (Dvoryanchikov et al. 2011). 


\section{Calcium imaging of isolated GAD65+ cells}

239 Type III cells are the only cells in the taste bud with voltage-gated calcium channels (Medler et 240 al., 2003; Romanov and Kolesnikov, 2006; Vandenbeuch et al., 2010), hence isolated Type III

241 cells are identifiable by robust calcium influx in response to depolarization with $\mathrm{KCl}$. As

242 expected, a subset of tdTomato negative cells exhibited calcium responses to $\mathrm{KCl}(\mathrm{CV}: 5 / 15, \mathrm{FF}$ :

243 3/7). However, a subset of tdTomato+ cells also had moderate to robust calcium responses to

$244 \mathrm{KCl}(\mathrm{CV}: 7 / 19, \mathrm{FF}: 3 / 13)$, consistent with reporter expression in Type III taste cells (Fig. 4).

245 Thus, these physiological data further support the argument that GAD65Cre does not 246 exclusively label Type I cells.

Chorda tympani responses elicited by blue light

When blue light is applied to the anterior tongue, robust chorda tympani responses are observed in GAD65Cre/ChR2 mice (Fig. 5). The amplitude of the responses to blue light (average ratio to baseline \pm sem: $3.07 \pm 0.41$ ) are larger than any other individual tastant used including $\mathrm{NH}_{4} \mathrm{Cl} 100 \mathrm{mM}(1.29 \pm 0.16)$, sucrose $(0.48 \pm 0.07), \mathrm{NaCl}(0.99 \pm 0.33)$, citric acid (0.45 $\pm 0.03)$, and quinine $(0.33 \pm 0.41)$ applied to the same region of the tongue. Responses to blue light are significantly different from all other tastants (One way ANOVA with Tukey's post-hoc; $F(1,5)=20.06, p<0.001)$. The large light responses are likely due to the large number of cells stimulated (including Type II and III cells). These data further support our contention that GAD65Cre drives ChR2 expression in multiple taste cell types.

\section{DISCUSSION}

262 The main goal of the present study was to characterize a commercially available mouse line that 263 expresses Cre recombinase from the Gad2 promoter (GAD65 protein), to evaluate use of this 264 allele to mark and/or manipulate gene expression in Type I taste cells. GAD65, encoded by the 265 Gad2 gene, is a Glutamate decarboxylase 2 enzyme which catalyzes production of gamma- 
aminobutyric acid (GABA) from L-glutamic acid. These mice have been used to examine the role of GABA and GAD65 in the brain where GAD65Cre-driven reporter expression was specific for GABA-expressing neurons with no mis-expression in other neuron types (Dergacheva et al., 2020; Quina et al., 2020; Whyland et al., 2020). This specific labeling is consistent with the observation that GABAergic neurons turn on Gad2 as they differentiate (Westmoreland et al., 2001). However, GAD65Cre activity has not been fully characterized in the peripheral taste

272 system, where taste cells are continuously generated throughout adult life (for a review, see

273 (Barlow, 2015)). Here, we crossed GAD65Cre mice with floxed tdTomato or channelrhodopsin

274 (ChR2) mice to identify and/or stimulate lineage traced cells. Although a majority of tdTomato or

275 ChR2 expressing cells also expressed the Type I cell-marker, NTPDase2, immunohistochemical 276 analyses demonstrate expression of the GAD65Cre-driven reporter in subsets of both Type II

277 and Type III cell types as well. This misexpression may be due to expression of Cre

278 recombinase in taste cell progenitors, if such progenitor cells transiently express GAD65. Thus,

279 if Cre recombinase is turned on in these progenitors, the progeny will continue to express the reporter, even though adult taste buds only express GAD65 in Type I cells.

281 Physiological studies also suggest that reporter expression is not limited to Type I taste cells.

282 First, calcium imaging experiments showed that a significant subset of isolated

283 GAD65Cre/tdTomato cells responded to $\mathrm{KCl}$ depolarization with increases in intracellular

284 calcium. The only adult taste cells known to exhibit voltage-gated calcium channels are the

285 Type III taste cells that utilize calcium dependent vesicular release of transmitter to activate 286 sensory afferent nerve fibers (Medler et al., 2003; DeFazio et al., 2006; Roberts et al., 2009;

287 Vandenbeuch et al., 2010). Although Dvoryanchikov et al (2011) reported Gad2 mRNA (coding

288 for GAD65 protein) expression in Type III adult taste cells (1/18 cells in CV) by single cell PCR,

289 our data show a much larger subset of isolated tdTomato cells with depolarization-evoked

290 calcium increases (7/19 in CV (Fisher's Exact two sided $P$ value $=0.042$ vs $1 / 18$ cells from

291 Dvoryanchikov et al); 3/13 in fungiform (not compared - Dvoryanchikov et al. did not examine

292 fungiform taste cells), suggesting that GAD65Cre likely drives reporter expression in some Type

293 III cell progenitors. Second, our chorda tympani nerve recordings show very large responses

294 when blue light is applied to the tongue -- responses larger than any evoked by a taste stimulus.

295 Although this does not rule out Type I cell stimulation activating nerve fibers, it does suggest

296 that ChR2 is driven in multiple cell types that all are activated by light.

297 In a recent study, (Baumer-Harrison et al., 2020) used the same constitutive GAD65Cre mice

298 crossed with floxed ChR2 mice to optogenetically stimulate taste buds. To examine reporter 
expression in taste buds, they used immunocytochemistry to label Type II (PLCb2) and Type III cells (Car4), and counted cells to determine co-localization with the reporter. No localization of ChR2 was observed in any cells that labeled with either Car4 or PLCb2, suggesting specificity of the reporter to Type I cells. Clearly these data differ from our results using the same Cre driver. We used different markers for Type II cells (GNAT vs PLCb2) and Type III cells (SNAP25 vs Car4), a different reporter for counting cells (tdTomato vs ChR2), and importantly, high-resolution confocal microscopy, and cross-sectional imaging of fungiform taste buds. We chose the tdTomato reporter for our studies as it is challenging to assess the degree of coexpression when comparing membrane-localized ChR2 to cytoplasmic immunoreactivity for Type II and III markers. With the large number of cells expressing ChR2, we were unable to say with certainty where individual cell boundaries began and ended. Thus, we used overlap of cytoplasmic tomato fluorescence with GFP and YFP expressed cytosolically in type II and III cells to determine with certainty whether GAD65Cre results in labeling of subsets of type II and III taste cells.

With regards to our cell profile counts, we may be underestimating the number of Type II or Type III cells that express tdTomato. For instance, in taste buds labeled with Gustducin (Figure 3) we are likely underestimating the number of Type II cells, as gustducin only identifies a subset of Type II cells (Clapp et al., 2001). Thus, some cells identified as tdTomato+ only could in fact be Gustducin-negative Type II cells that also express tdTomato. In taste buds of GAD65Cre/tdTomato/TRPM5GFP/PKD2L1YFP mice, while we are likely identifying nearly all Type II cells with TRPM5GFP, we may be underestimating the number of Type III cells. While PKD2L1YFP reliably identifies the majority of Type III cells in all taste fields (Wilson et al., 2017), the YFP we are detecting is tethered to the PKD2L1 protein which is predominantly localized in apical regions of Type III cells. Cell profiles were counted in 2-3 planes of the taste bud, spaced $\sim 10$ um apart. Thus, in more basal planes, PDK2L1YFP is not a reliable identifier of Type III cells. Further, overall, we could be underrepresenting the cell counts given the high thresholds we are using for expression, especially the fungiform taste buds where $\sim 14 \%$ of profiles analyzed were below threshold in every channel. Thus, we predict that the GAD65cre is expressed in a higher percentage of Type II or Type III cells than calculated here. 
amiloride-sensitive salt responses were mediated by Type I taste cells. Our data would argue that while Type I cells were likely stimulated in their experiments, other cell types were also stimulated and it is not clear which cells were actually responsible for amiloride-sensitive salt taste. Another recent study also sheds doubt on Type I cells mediating amiloride-sensitive salt taste. (Nomura et al., 2020) showed that while amiloride-sensitive currents were present in a subset of Type I taste cells, validated by expression of NTPDase2, they did not signal to the nervous system. Instead, a subset of cells co-expressing the amiloride-sensitive Na channel, the ATP release channel CALHM1, and voltage-gated sodium channels were required for amiloride-sensitive salt taste chorda tympani responses. Type I cells express neither Calhm1 (Taruno et al., 2013) nor voltage-gated sodium channels (Medler et al., 2003; Romanov and

341 Kolesnikov, 2006; Vandenbeuch et al., 2010). Nomura et al., suggest that amiloride-sensitive

342 salt taste is likely mediated by a unique cell type, distinct from Type II or Type III cells, which

343 has also been suggested by others (Chandrashekar et al., 2010; Roebber et al., 2019). It is

344 certainly possible that GAD65Cre drives expression in this unique cell type or progenitors that give rise to this specific taste cell type, which is responsible for sodium-specific salt taste but it is likely not Type I cells.

In summary our data indicate that GAD65Cre activity drives expression of reporter alleles in type II and III taste cells in addition to type I cells, perhaps due to transient expression in taste progenitors. Thus, even though the majority of GAD65Cre/tdTomato+ cells appear to be Type I cells, results using this mouse line to study Type I cell function specifically must be interpreted cautiously

\section{CONFLICT OF INTERESTS}




\section{ACKNOWLEDGEMENTS}

359 The authors want to thank Drs. Thomas Finger and Linda Barlow for helpful discussions during 360 the course of this study. 
REFERENCES

364

365

366

367

368

369

370

371

372

373

374

375

376

377

378

379

380

381

382

383

384

385

386

387

388

389

390

391

392

393

394

395

396

397

398

399

400

401

Barlow, L.A. 2015. Progress and renewal in gustation: new insights into taste bud development. Development. 142:3620-3629.

Bartel, D.L., Sullivan, S.L., Lavoie, É.G., Sévigny, J., and Finger, T.E. 2006. Nucleoside triphosphate diphosphohydrolase-2 is the ecto-ATPase of type I cells in taste buds. J Comp Neurol. 497:1-12.

Baumer-Harrison, C., Raymond, M.A., Myers, T.A., Sussman, K.M., Rynberg, S.T., Ugartechea, A.P., Lauterbach, D., Mast, T.G., and Breza, J.M. 2020. Optogenetic Stimulation of Type I GAD65+ Cells in Taste Buds Activates Gustatory Neurons and Drives Appetitive Licking Behavior in Sodium-Depleted Mice. J Neurosci. 40:7795-7810.

Béhé, P., DeSimone, J.A., Avenet, P., and Lindemann, B. 1990. Membrane currents in taste cells of the rat fungiform papilla. Evidence for two types of $\mathrm{Ca}$ currents and inhibition of $\mathrm{K}$ currents by saccharin. J Gen Physiol. 96:1061-1084.

Castillo, D., Seidel, K., Salcedo, E., Ahn, C., Sauvage, F.J. de, Klein, O.D., and Barlow, L.A. 2014. Induction of ectopic taste buds by SHH reveals the competency and plasticity of adult lingual epithelium. Development. 141:2993-3002.

Chandrashekar, J., Kuhn, C., Oka, Y., Yarmolinsky, D.A., Hummler, E., Ryba, N.J.P., and Zuker, C.S. 2010. The cells and peripheral representation of sodium taste in mice. Nature. 464:297-301.

Chang, R.B., Waters, H., and Liman, E.R. 2010. A proton current drives action potentials in genetically identified sour taste cells. Proc Natl Acad Sci. 107:22320-22325.

Clapp, T.R., Stone, L.M., Margolskee, R.F., and Kinnamon, S.C. 2001. Immunocytochemical evidence for co-expression of Type III IP3 receptor with signaling components of bitter taste transduction. BMC Neurosci. 2:6.

Clapp, T.R., Medler, K.F., Damak, S., Margolskee, R.F., and Kinnamon, S.C. 2006. Mouse taste cells with $\mathrm{G}$ protein-coupled taste receptors lack voltage-gated calcium channels and SNAP-25. BMC Biol. 4:7.

Corson, J.A., and Erisir, A. 2013. Monosynaptic convergence of chorda tympani and glossopharyngeal afferents onto ascending relay neurons in the nucleus of the solitary tract: A high-resolution confocal and correlative electron microscopy approach. J Comp Neurol. 521:2907-2926.

DeFazio, R.A., Dvoryanchikov, G., Maruyama, Y., Kim, J.W., Pereira, E., Roper, S.D., and Chaudhari, N. 2006. Separate Populations of Receptor Cells and Presynaptic Cells in Mouse Taste Buds. J Neurosci. 26:3971-3980.

Dergacheva, O., Fleury-Curado, T., Polotsky, V.Y., Kay, M., Jain, V., and Mendelowitz, D. 2020. GABA and glycine neurons from the ventral medullary region inhibit hypoglossal motoneurons. Sleep. 43.

Dvoryanchikov, G., Huang, Y.A., Barro-Soria, R., Chaudhari, N., and Roper, S.D. 2011. GABA, Its Receptors, and GABAergic Inhibition in Mouse Taste Buds. J Neurosci. 31:5782-5791. 
402

403

404

405

406

407

408

409

410

411

412

413

414

415

416

417

418

419

420

421

422

423

424

425

426

427

428

429

430

431

432

433

434

435

436

437

438

439

440

Gaillard, D., Xu, M., Liu, F., Millar, S.E., and Barlow, L.A. 2015. $\beta$-Catenin Signaling Biases Multipotent Lingual Epithelial Progenitors to Differentiate and Acquire Specific Taste Cell Fates. PLOS Genet. 11:e1005208.

Huang, A.Y., and Wu, S.Y. 2018. Substance $P$ as a putative efferent transmitter mediates GABAergic inhibition in mouse taste buds. Br J Pharmacol. 175:1039-1053.

Larson, E.D., Vandenbeuch, A., Anderson, C.B., and Kinnamon, S.C. 2020. Function, Innervation, and Neurotransmitter Signaling in Mice Lacking Type-II Taste Cells. ENeuro. 7.

Medler, K.F., Margolskee, R.F., and Kinnamon, S.C. 2003. Electrophysiological

Characterization of Voltage-Gated Currents in Defined Taste Cell Types of Mice. J Neurosci. 23:2608-2617.

Nomura, K., Nakanishi, M., Ishidate, F., Iwata, K., and Taruno, A. 2020. All-Electrical Ca2+Independent Signal Transduction Mediates Attractive Sodium Taste in Taste Buds. Neuron. 106:816-829.e6.

Quina, L.A., Walker, A., Morton, G., Han, V., and Turner, E.E. 2020. GAD2 Expression Defines a Class of Excitatory Lateral Habenula Neurons in Mice that Project to the Raphe and Pontine Tegmentum. ENeuro. 7.

R Core Team. 2019. R: A Language and Environment for Statistical Computing. Vienna, Austria: R Foundation for Statistical Computing.

Roberts, C.D., Dvoryanchikov, G., Roper, S.D., and Chaudhari, N. 2009. Interaction between the second messengers cAMP and Ca2+ in mouse presynaptic taste cells. J Physiol. 587:16571668.

Roebber, J.K., Roper, S.D., and Chaudhari, N. 2019. The Role of the Anion in Salt (NaCl) Detection by Mouse Taste Buds. J Neurosci. 39:6224-6232.

Romanov, R.A., and Kolesnikov, S.S. 2006. Electrophysiologically identified subpopulations of taste bud cells. Neurosci Lett. 395:249-254.

Romanov, R.A., Lasher, R.S., High, B., Savidge, L.E., Lawson, A., Rogachevskaja, O.A., Zhao, H., Rogachevsky, V.V., Bystrova, M.F., Churbanov, G.D., et al. 2018. Chemical synapses without synaptic vesicles: Purinergic neurotransmission through a CALHM1 channelmitochondrial signaling complex. Sci Signal. 11:eaao1815.

Roper, S.D., and Chaudhari, N. 2017. Taste buds: cells, signals and synapses. Nat Rev Neurosci. 18:485-497.

Sinclair, M.S., Perea-Martinez, I., Dvoryanchikov, G., Yoshida, M., Nishimori, K., Roper, S.D., and Chaudhari, N. 2010. Oxytocin Signaling in Mouse Taste Buds. PLOS ONE. 5:e11980.

Stratford, J., Larson, E., Yang, R., Salcedo, E., and Finger, T. 2017. 5-HT3a-driven GFP delineates Gustatory Fibers innervating Sour-responsive Taste Cells: A Labeled Line for Sour Taste? J Comp Neurol. 525:2358-2375.

Taniguchi, H., He, M., Wu, P., Kim, S., Paik, R., Sugino, K., Kvitsani, D., Fu, Y., Lu, J., Lin, Y., et al. 2011. A Resource of Cre Driver Lines for Genetic Targeting of GABAergic Neurons in Cerebral Cortex. Neuron. 71:995-1013. 
Taruno, A., Vingtdeux, V., Ohmoto, M., Ma, Z., Dvoryanchikov, G., Li, A., Adrien, L., Zhao, H., Leung, S., Abernethy, M., et al. 2013. CALHM1 ion channel mediates purinergic neurotransmission of sweet, bitter and umami tastes. Nature. 495:223-226.

Vandenbeuch, A., Anderson, C.B., Parnes, J., Enjyoji, K., Robson, S.C., Finger, T.E., and Kinnamon, S.C. 2013. Role of the ectonucleotidase NTPDase2 in taste bud function. Proc Natl

447 Vandenbeuch, A., Clapp, T.R., and Kinnamon, S.C. 2008. Amiloride-sensitive channels in type I 448 fungiform taste cells in mouse. BMC Neurosci. 9:1.

449 Vandenbeuch, A., Zorec, R., and Kinnamon, S.C. 2010. Capacitance Measurements of Regulated Exocytosis in Mouse Taste Cells. J Neurosci. 30:14695-14701.

451 Vandenbeuch, A., Larson, E.D., Anderson, C.B., Smith, S.A., Ford, A.P., Finger, T.E., and 452 Kinnamon, S.C. 2015. Postsynaptic P2X3-containing receptors in gustatory nerve fibres 453 mediate responses to all taste qualities in mice. J Physiol. 593:1113-1125.

454 Westmoreland, J.J., Hancock, C.R., and Condie, B.G. 2001. Neuronal Development of 455 Embryonic Stem Cells: A Model of GABAergic Neuron Differentiation. Biochemical and 456 Biophysical Research Communications. 284:674-680.

457 Whyland, K.L., Slusarczyk, A.S., and Bickford, M.E. 2020. GABAergic cell types in the 458 superficial layers of the mouse superior colliculus. J Comp Neurol. 528:308-320.

459 Wickham, H. 2016. ggplot2: Elegant Graphics for Data Analysis. Springer-Verlag New York.

460 Wilson, C.E., Finger, T.E., and Kinnamon, S.C. 2017. Type III Cells in Anterior Taste Fields Are 461 More Immunohistochemically Diverse Than Those of Posterior Taste Fields in Mice. Chem 462 Senses. 42:759-767.

Yang, R., Dzowo, Y.K., Wilson, C.E., Russell, R.L., Kidd, G.J., Salcedo, E., Lasher, R.S., Kinnamon, J.C., and Finger, T.E. 2020. Three-dimensional reconstructions of mouse circumvallate taste buds using serial blockface scanning electron microscopy: I. Cell types and 
$470 \quad$ Figure 1.

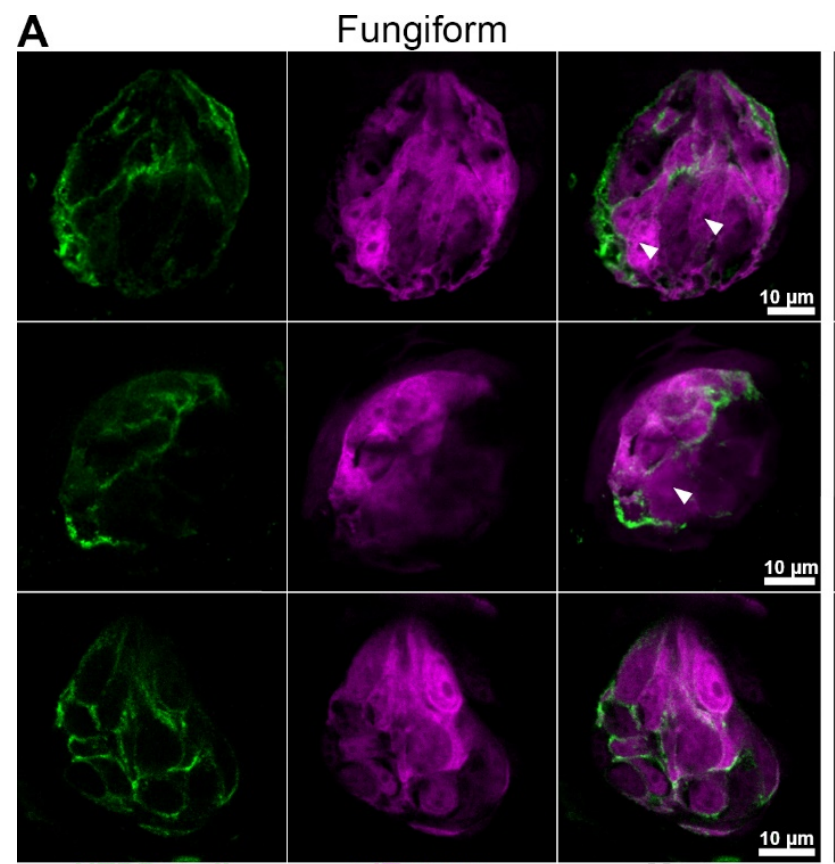

NTPDasell

tdTomato

Merge
$10 \mathrm{~mm}$

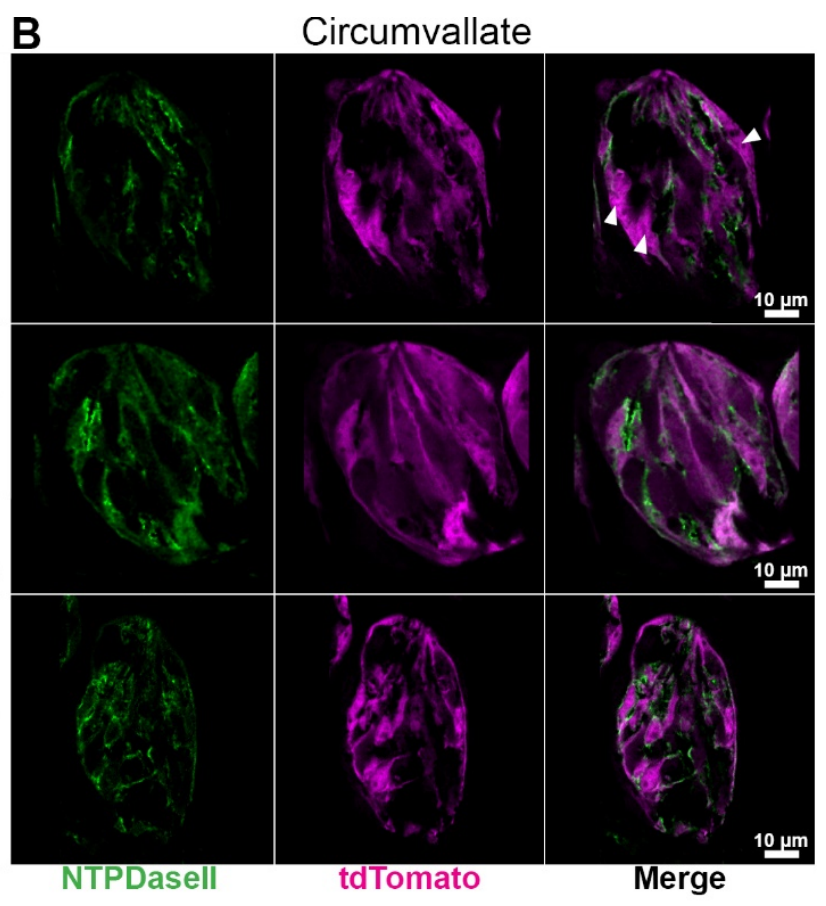

4732 column figure, width 7.09 inches

474 Confocal images of fungiform (A) and circumvallate (B) tastebuds from GAD65Cre/tdTomato 475 mice. Images are single optical planes. Six representative taste buds sectioned longitudinally

476 show NTPDase2 immunoreactivity (green) and endogenous tdTomato fluorescence (magenta)

477 in the taste bud. NTPDase2 immunoreactivity is mostly associated with tdTomato fluorescence.

478 Arrowheads show areas of tdTomato not associated with strong membrane-localized

479 NTPDase2 immunoreactivity. Scale bars, $10 \mu \mathrm{m}$. 


\section{$482 \quad$ Figure 2.}

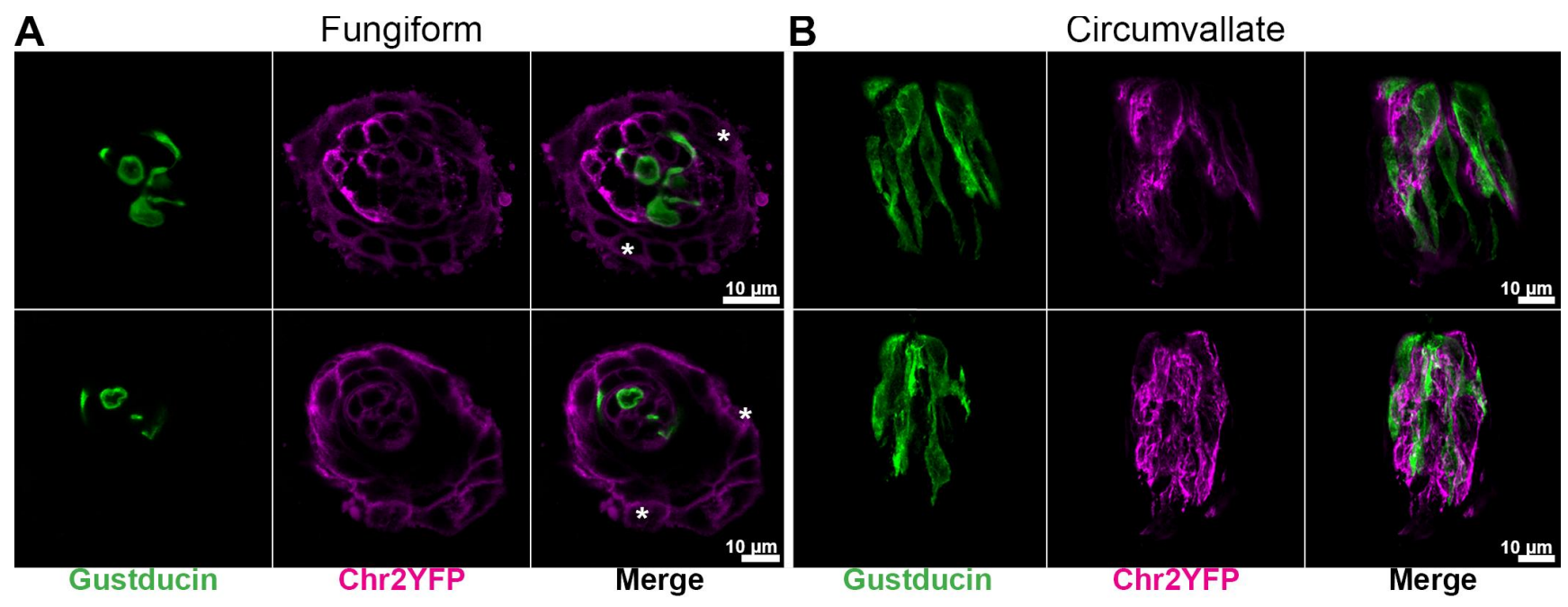

486 Confocal images of whole-mount fungiform (A) and longitudinally sliced circumvallate (B) taste 487 buds from GAD65Cre/ChR2. Fungiform images are single optical planes while CV images are a 488 Z-stacks of 2 axial planes spaced $0.75 \mu \mathrm{m}$ apart. In many GAD65Cre/ChR2 fungiform taste 489 buds, a ring of ChR2+ cells was observed outside the taste bud (asterisks). The identity of these 490 cells is unclear. Additionally, while ChR2 was observed surrounding Gustducin+ cells, it is 491 unclear if the signals are in the same cell, given the proximity of cells with one another in taste 492 buds. 


\section{$495 \quad$ Figure 3}

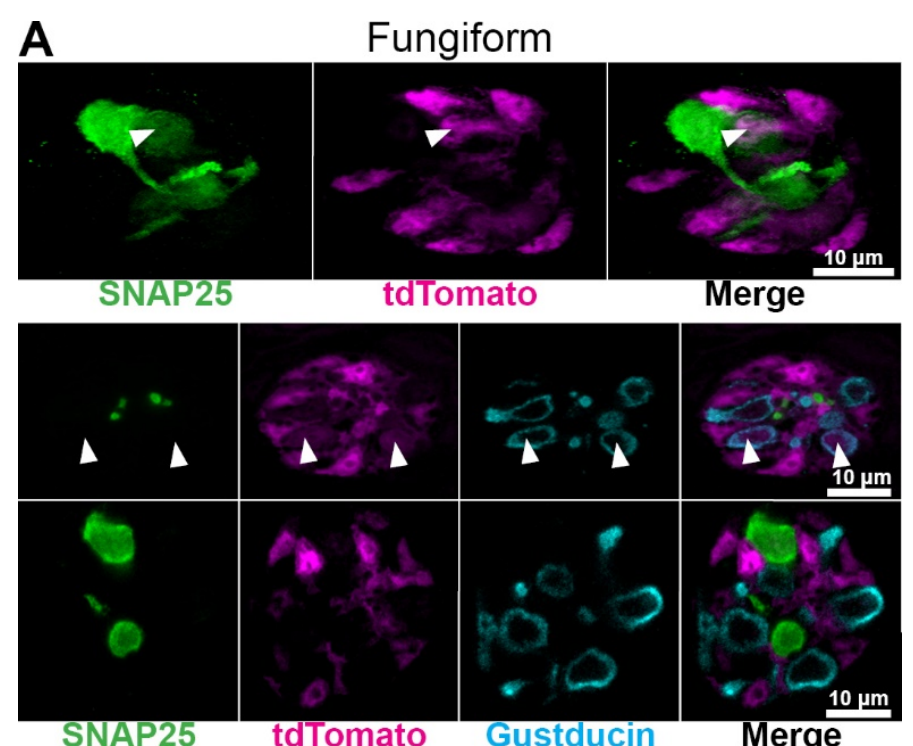

B
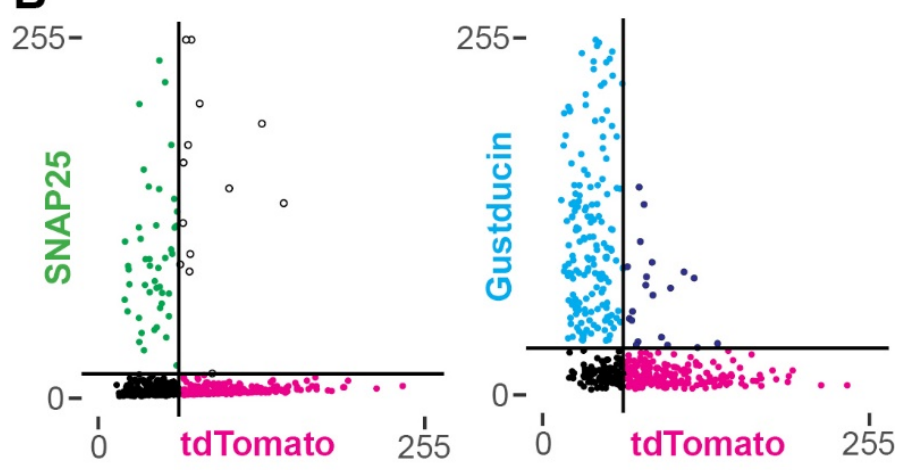

4971 column figure, width 3.54 inches

498 A. Confocal images of whole-mount fungiform taste buds from GAD65Cre/tdTomato mice.

499 Fungiform taste buds are imaged in cross section. Images are single optical planes. Type III

500 cells are identified by SNAP25 immunoreactivity (green) and a subset of type II cells are

501 identified by Gustducin immunoreactivity (cyan). Arrowheads indicate tdTomato fluorescence in

502 either a Type II or Type III cell. Images were background-subtracted and scaled using the same

503 method for quantification (see methods). B. Quantification of cell profile fluorescence intensities.

504 Cell profiles were measured in optical sections to assess the degree of overlapping signals.

505 Horizontal and vertical black lines show the calculated threshold levels of 2 standard deviations

506 above background. Black points are those below threshold in both channels. Points in the upper

507 right quadrant are those found above-threshold in both channels. 
bioRxiv preprint doi: https://doi.org/10.1101/2021.01.14.426727; this version posted January 17, 2021. The copyright holder for this preprint (which was not certified by peer review) is the author/funder. All rights reserved. No reuse allowed without permission. 


\section{$509 \quad$ Figure 4.}

A

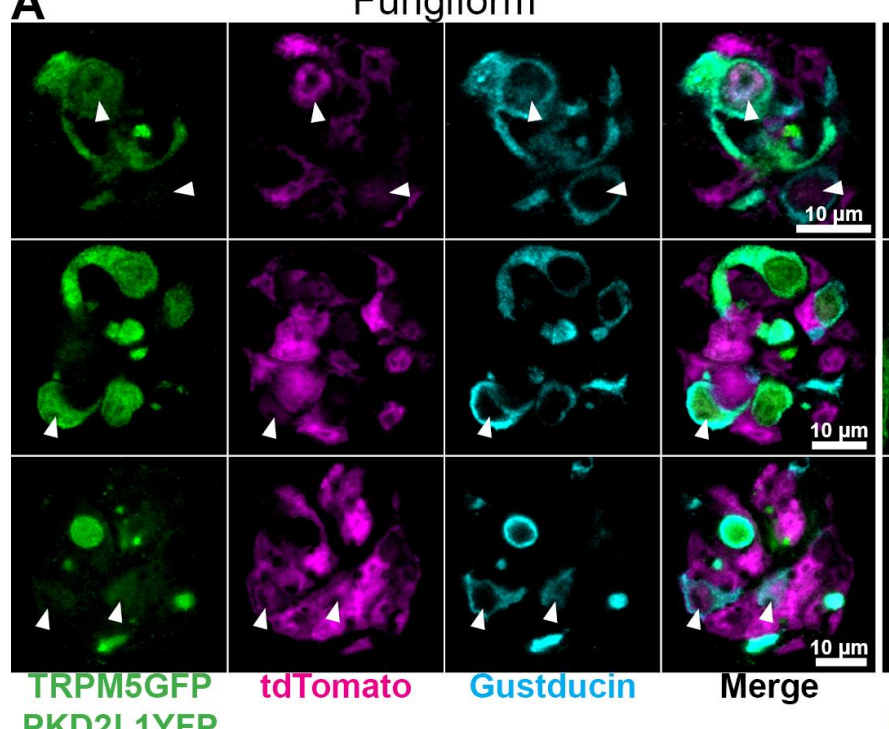

PKD2L1YFP

B
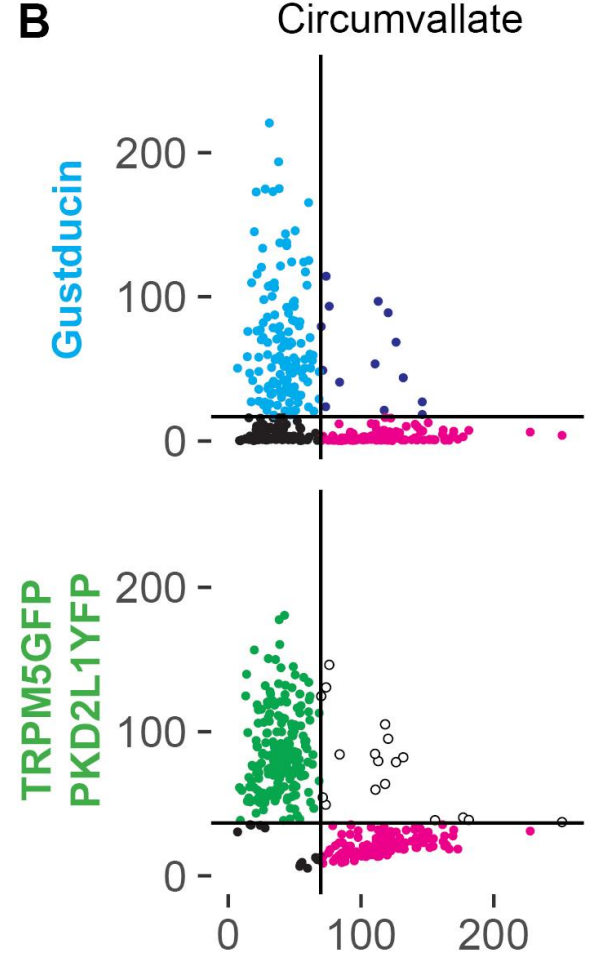

510

511

512

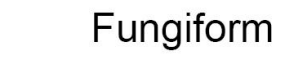

Circumvallate

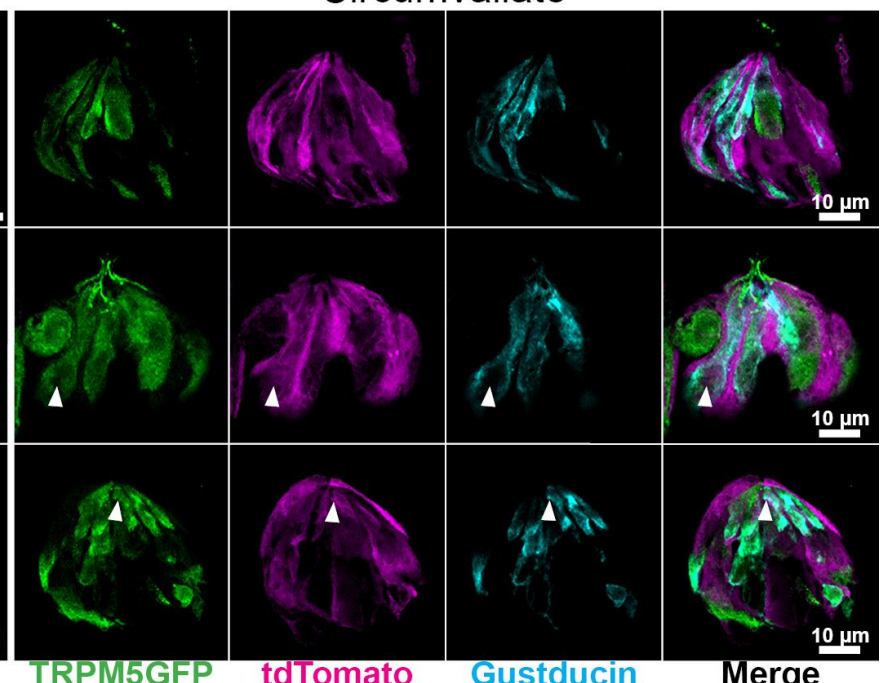

Foliate

Soft Palate
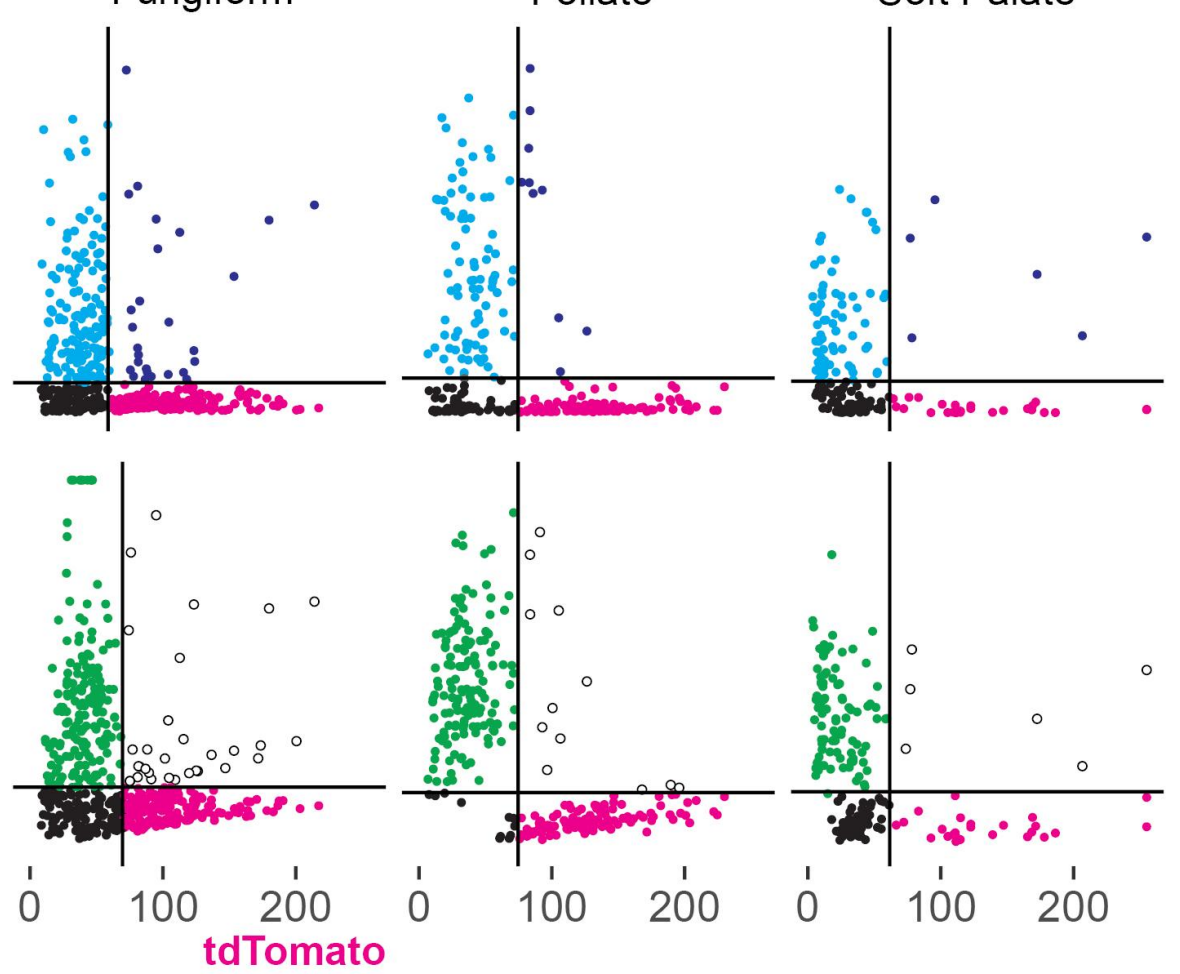

100200

100200 tdTomato 
513 Confocal images of whole-mount fungiform taste buds from

514 GAD65Cre/tdTomato/TRPM5GFP/PKD2L1YFP mice. Taste buds are imaged in cross section.

515 Images are single optical planes. Type II and III cells are visualized with GFP immunoreactivity

516 as the antibody reacts with both GFP and YFP. A subset of type II cells is visualized by

517 Gustducin immunoreactivity. tdTomato fluorescence in a Type II/III cell is observed in at least 1

518 cell of each taste bud (arrowheads). B. Quantification of cell profile mean fluorescence

519 intensities. Cell profiles were measured in optical sections to assess the degree of overlapping

520 signals. Horizontal and vertical black lines show the calculated threshold levels of 2 standard

521 deviations above background. Black points are those below threshold in both channels. Points

522 in the upper right quadrant are those found above-threshold in both channels.

523

524

525 


\section{$526 \quad$ Figure 5.}
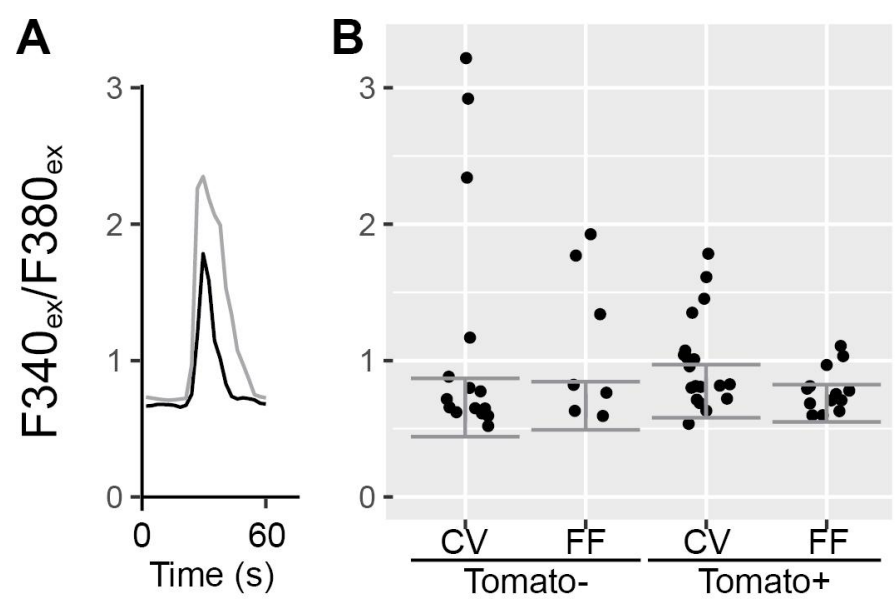

5281 column figure, width 3.54 inches

529 Fura2 calcium imaging of isolated GAD65Cre/tdTomato cells. tdTomato positive and negative

530 taste cells were loaded with Fura2 and exposed to depolarizing concentrations of $\mathrm{KCl}$ to test for

531 the presence of voltage gated calcium channels. Responses are displayed as a ratio of

532 emission at 510 from excitations at 340 and $380 \mathrm{~nm}$. A. Two example cells showing a response

533 to $\mathrm{KCl}$ (black: tdTomato positive, grey: tdTomato negative). B. Responses of individual cells.

534 Horizontal position of each dot within each group is arbitrary: dots are jittered to visualize

535 overlapping points. Horizontal error bars depict 2 standard deviations above and below median

536 of baseline values across all cells per group. Responses over median plus 2 standard

537 deviations were considered positive.

538

539 


\section{$541 \quad$ Figure 6.}
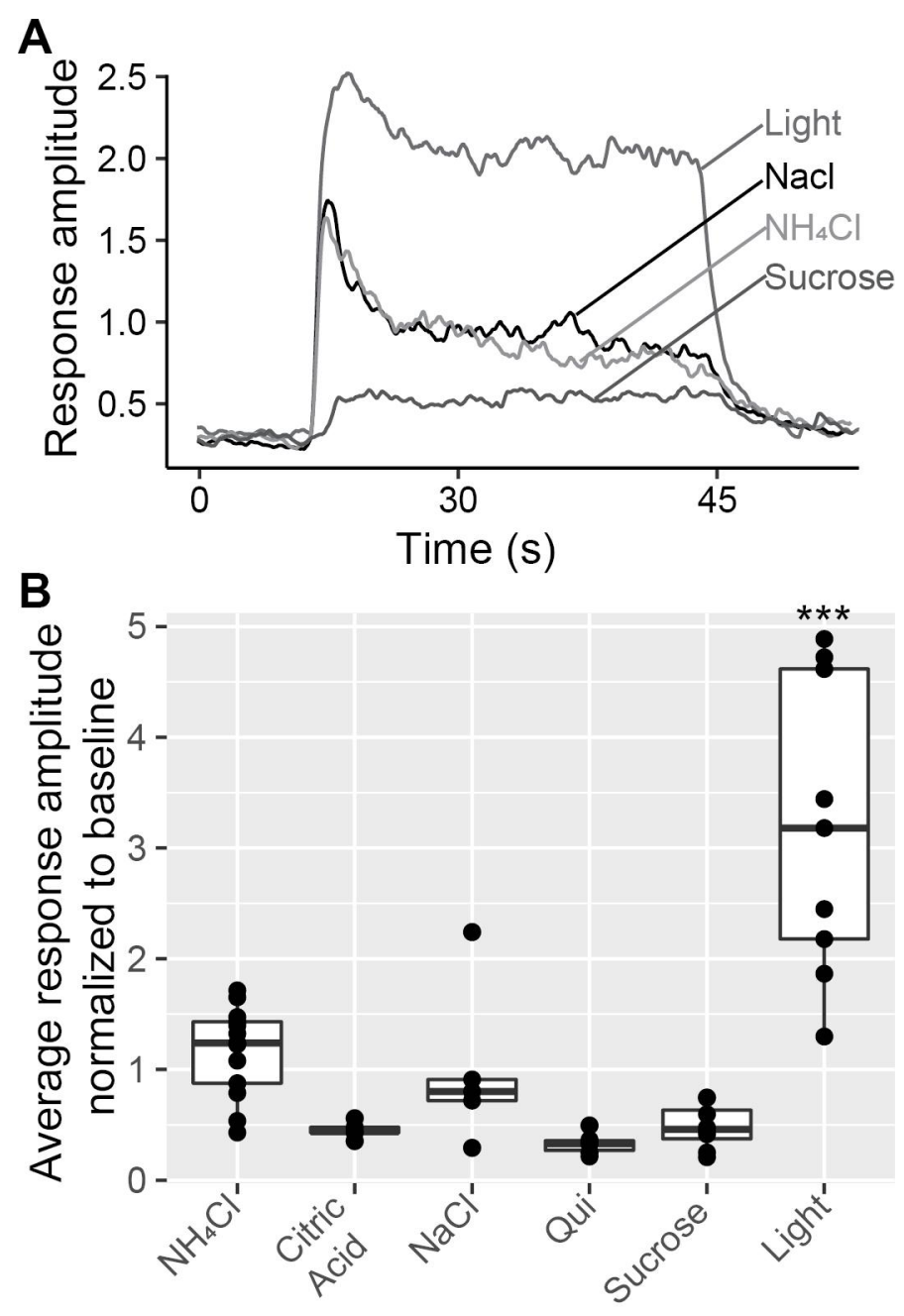

5431 column figure, width 3.54 inches

544 Chorda tympani nerve recordings in GAD65Cre/ChR2 mice. A: Representative integrated nerve 545 recordings in response to light $(5 \mathrm{~Hz}), \mathrm{NaCl} 100 \mathrm{mM}, \mathrm{NH}_{4} \mathrm{Cl} 100 \mathrm{mM}$, and sucrose $500 \mathrm{mM}$. B:

546 Average chorda tympani responses normalized to baseline. Dots represent individual animals

547 and the box plot demonstrates distribution of data. ${ }^{* * *}$ indicates significance at $p<0.001$

548 compared to all other tastants by One way ANOVA with Tukey's post-hoc. No differences

549 among tastants were observed. 


\section{${ }_{553}$ TABLES}

554 Table 1

\begin{tabular}{|l|l|l|l|}
\hline Name & Jax stock & Jax full name & background \\
\hline GAD65Cre & 010802 & Gad2-IRES-Cre & C57BL/6J \\
\hline tdTomato & 007909 & Ai9(RCL-tdT) & C57BL/6J \\
\hline ChR2 & 024109 & Ai32(RCL-ChR2(H134R)/EYFP) & C57BL/6J \\
\hline TRPM5GFP & n/a & n/a & C57BL/6J \\
\hline PKD2L1YFP & n/a & n/a & C57BL/6J \\
\hline
\end{tabular}

556 Mouse lines used in this study. TRPM5GFP mice are described in (Clapp et al., 2006) and 557 PKD2L1YFP mice are described in (Chang et al., 2010). 
559 Table 2

\begin{tabular}{|c|c|c|c|c|}
\hline Antiserum & Company & $\begin{array}{l}\text { Catalog } \\
\text { Number }\end{array}$ & Dilution & RRID \\
\hline $\begin{array}{l}\text { Chicken } \\
\text { polyclonal anti- } \\
\text { GFP }\end{array}$ & Aves Lab & $\begin{array}{l}\text { GFP- } \\
1020\end{array}$ & $1: 2000$ & AB_10000240 \\
\hline $\begin{array}{l}\text { Goat polyclonal } \\
\text { anti- Gustducin }\end{array}$ & Aviva Systems Bio & $\begin{array}{l}\text { NC95105 } \\
98\end{array}$ & $1: 500$ & $A B \_10882823$ \\
\hline $\begin{array}{l}\text { Rabbit polyclonal } \\
\text { anti-SNAP25 }\end{array}$ & Sigma & S9684 & $1: 500$ & AB_261576 \\
\hline $\begin{array}{l}\text { Rabbit polyclonal } \\
\text { anti-NTPDasell }\end{array}$ & $\begin{array}{l}\text { J. Sevigny, Laval } \\
\text { University; Quebec; } \\
\text { Canada, Bartel et } \\
\text { al. } 2006\end{array}$ & & $1: 500$ & $A B \_2314986$ \\
\hline
\end{tabular}

561 List of primary antisera used in this study. 


\section{Table 3}

\begin{tabular}{|c|c|c|c|c|}
\hline Channel & CV & FF & Fol & SP \\
\hline Gustducin only & $3(0.8 \%)$ & $5(1.0 \%)$ & 0 & $2(1.1 \%)$ \\
\hline tdTomato only & $130(35.9 \%)$ & $133(26.0 \%)$ & $101(37.0 \%)$ & $24(12.8 \%)$ \\
\hline $\begin{array}{c}\text { TRPM5GFP/PKD2L1YFP } \\
\text { only }\end{array}$ & $65(18.0 \%)$ & $74(14.5 \%)$ & $49(17.9 \%)$ & $21(11.2 \%)$ \\
\hline $\begin{array}{c}\text { Gustducin + } \\
\text { TRPM5GFP/PKD2L1YFP }\end{array}$ & $133(36.7 \%)$ & $156(30.5 \%)$ & $87(33.7 \%)$ & $77(41.2 \%)$ \\
\hline $\begin{array}{c}\text { Gustducin + tdTomato } \\
\text { TRPM5GFP/PKD2L1YFP }\end{array}$ & $3(0.9 \%)$ & $3(0.6 \%)$ & $2(0.8 \%)$ & 0 \\
\hline $\begin{array}{c}\text { tdTomato + } \\
\text { GRPStucin + tdTomato + }\end{array}$ & $11(3.0 \%)$ & $29(5.7 \%)$ & $8(3.1 \%)$ & $6(3.2 \%)$ \\
\hline $\begin{array}{c}\text { TRPFP/PKD2L1YFP } \\
\text { sub-threshold }\end{array}$ & $10(2.8 \%)$ & $74(14.5 \%)$ & $7(2.7 \%)$ & $56(29.9 \%)$ \\
\hline
\end{tabular}

568 Summary of quantification in Figure 4. Counts are number of ROIs displaying above-threshold 569 pixel values for each channel or channel combination. Percentages are reflective of percent 570 ROls from each taste field. Each cell profile is binned according to channel, thus a single cell 571 profile is counted only once. 